\title{
The metallicity of the open cluster Tombaugh $2^{\star}$
}

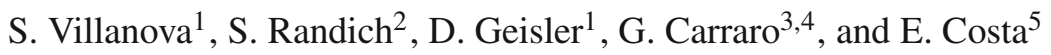 \\ 1 Universidad de Concepción, Departamento de Astronomia, Casilla 160-C, Concepción, Chile \\ e-mail: [svillanova; dgeisler] @astro-udec.cl \\ 2 INAF - Osservatorio Astrofisico di Arcetri, Largo E. Fermi 5, 50125 Firenze, Italy \\ e-mail: randich@arcetri.astro.it \\ 3 ESO, Alonso de Cordova 3107, Vitacura, Santiago, Chile \\ e-mail: gcarraro@eso.org \\ 4 Dipartimento di Astronomia, Universitá di Padova, Vicolo Osservatorio 3, 35122, Padova, Italy \\ 5 Departamento de Astronomía, Universidad de Chile, Casilla 36-D, Santiago, Chile \\ e-mail: costa@das.uchile.cl
}

Received 7 September 2009 / Accepted 5 November 2009

\begin{abstract}
Context. Open clusters are excellent tracers of the structure, kinematics, and chemical evolution of the disk and a wealth of information can be derived from the spectra of their constituent stars.

Aims. We investigate the nature of the chemical composition of the outer disk open cluster Tombaugh 2 . This has been suggested to be a member of the GASS/Mon substructure, and a recent study by Frinchaboy et al. (2008) suggested that this was a unique open cluster in possessing an intrinsic metal abundance dispersion. We aim to investigate such claims.

Methods. High resolution VLT+GIRAFFE spectra in the optical are obtained and analyzed for a number of stars in the Tombaugh 2 field, together with independent $U B V I_{\mathrm{C}}$ photometry. Radial velocities and position in the color-magnitude diagram are used to assess cluster membership. The spectra, together with input atmospheric parameters and model atmospheres, are used to determine detailed chemical abundances for a variety of elements in 13 members with good spectra.

Results. We find the mean metallicity to be $[\mathrm{Fe} / \mathrm{H}]=-0.31 \pm 0.02$ with no evidence for an intrinsic abundance dispersion, in contrary to the recent results of Frinchaboy et al. (2008, MNRAS, 391, 39). We find Ca and Ba to be slightly enhanced, while Ni and Sc are solar. The r-process element Eu was found to be enhanced, giving an average $[\mathrm{Eu} / \mathrm{Ba}]=+0.17$. The $\mathrm{Li}$ abundance decreases with $T_{\text {eff }}$ on the upper giant branch and maintains a low level for red clump stars. The mean metallicity we derive agrees well with that expected from the radial abundance gradient in the disk for a cluster at its Galactocentric distance.

Conclusions. Tombaugh 2 is found to have abundances as expected from its Galactocentric distance and no evidence for any intrinsic metallicity dispersion. The surprising result found by Frinchaboy et al. (2008), which is the presence of two distinct abundance groups within the cluster, implying either a completely unique open cluster with an intrinsic metallicity spread or a very unlikely superposition of a cold stellar stream and a very distant open cluster, is not supported by our new result.
\end{abstract}

Key words. Galaxy: disk - open clusters and associations: general - open clusters and associations: individual: Tombaugh 2 Galaxy: structure

\section{Introduction}

One of the many regions in our Galaxy for which we lack detailed knowledge is the outer disk. How and when was it formed? By an outside-in or inside-out process (Chiappini et al. 2001)? What is the nature of the metallicity gradient? Does it show a constant slope with Galactocentric distance, or is there a leveling out in the outer disk (Twarog et al. 1997; Carraro et al. 2004 , 2007; Magrini et al. 2009)? What if any is the role of mergers?

A particularly intriguing development in recent years has been the suggestion that there is a merger remnant lying near the Galactic plane. The structure was first identified as the Monoceros stream (Mon; Newberg et al. 2002; Ibata et al. 2003; Yanny et al. 2003), also known as the Galactic anticenter stellar structure (GASS; Rocha-Pinto et al. 2003; Crane et al. 2003). GASS/Mon was discovered as an overdensity of stars near the Galactic plane that seems to wrap around the outer parts of the

* Based on observations collected at ESO-VLT, Paranal Observatory, Chile, Program numbers 076.D-0220(A).
Galactic disk and is frequently explained as tidal debris from the disruption of a dwarf galaxy on a low inclination orbit (e.g., Crane et al. 2003; Yanny et al. 2003; Peñarrubia et al. 2005).

Further arguments have been made that the reputed dwarf galaxy in Canis Major (CMa; Bellazzini et al. 2004) is the progenitor of the Mon/GASS structure (Martin et al. 2004). However, the nature and/or reality of the proposed Mon "tidal debris stream" and the CMa overdensity have been called into question and are currently a matter of great debate. Momany (2004; 2006) have argued that much of the observed stellar overdensity associated with Mon - and particularly all of that associated with $\mathrm{CMa}$ (at $l \sim 240^{\circ}$ ) - is due to the warping and flaring of the Galactic disk, and that no "extra-Galactic" component is needed to account for the apparent overdensities in the third quadrant. The presence of "blue plume" stars in this part of the sky has been used to argue further for the presence of a dwarf galaxy nucleus in CMa (Bellazzini et al. 2004; Martínez-Delgado et al. 2005; Dinescu et al. 2005; Butler et al. 2007). However, these young stars have also been more 
prosaically attributed to the presence of previously unknown features of spiral arm structure (Carraro et al. 2005; Moitinho et al. 2006).

Open clusters have traditionally played a critical role in the study of the structure, kinematics, formation and chemical evolution of the disk. For this reason alone, the old outer Galactic disk open cluster Tombaugh 2 (To2; at Galactic coordinates $\left.[l, b]=[232.8,-6.9]^{\circ}\right)$, located at a Galactocentric distance of $\sim 15 \mathrm{kpc}$ and $700 \mathrm{pc}$ below the Galactic plane, is worthy of study and was indeed the subject of several previous metallicity investigations. Brown et al. (1996) obtained high resolution spectra of three stars, finding $[\mathrm{Fe} / \mathrm{H}]=-0.4 \pm 0.25$, while Friel et al. (2002) derived $[\mathrm{Fe} / \mathrm{H}]-0.44 \pm 0.09$ from much lower resolution spectra of 12 members. Both of these studies were conducted with the CTIO 4 m telescope. Subsequently, Frinchaboy et al. (2004) suggested that To2 may possibly be associated with Mon/GASS as well as with CMa (Bellazzini et al. 2004). Note that its Galactic longitude is very close to that of the CMa overdensity. This provided a strong additional incentive for a followup study of its chemistry and kinematics in greater detail with a larger telescope, as even the giants are faint due to its distance of over $13 \mathrm{kpc}$. Frinchaboy et al. (2008 - hereafter F08) used this motivation to obtain VLT/FLAMES spectra (both with UVES at high resolution and with GIRAFFE at somewhat lower resolution). They were able to derive velocities and detailed abundances of a number of elements for $18 \mathrm{To} 2$ cluster members. Their most surprising result was an apparent large spread in metallicity: $\Delta[\mathrm{Fe} / \mathrm{H}]>0.2$. They were unable to account for this spread given their observational errors and presented a number of possible scenarios, including the likelihood that To2 possessed an intrinsic metallicity spread. They argued for the possible presence of two populations in the cluster, distinguished by their different mean chemical characteristics - with a metal-rich, Ti-normal group and a metal-poor, Ti-enhanced group, namely $(\langle[\mathrm{Fe} / \mathrm{H}]\rangle$, $\langle[\mathrm{Ti} / \mathrm{Fe}]\rangle) \sim(-0.06,+0.02)$ and $(-0.28,+0.36)$. The more metalpoor group appeared more centrally concentrated, and they suggested that this group represented the true To 2 clusters stars, and the metal-rich population was an overlapping, and kinematically associated, but "cold" stellar stream.

If true, this would be the first such metallicity spread uncovered in an open cluster, and/or the first evidence of multiple populations in a cluster of this age (about 2 Gyr) in the Galaxy, making To2 an extremely intriguing object. Although multiple populations within globular clusters are now known to exist, these clusters are all much more massive than an open cluster such as To2, as expected if this phenomenon is due to a cluster being massive enough to retain ejecta from a first generation of stars in order to make a second, chemically enriched generation. To date, the F08 study is the only one which suggests multiple populations in an open cluster. However, as F08 pointed out, further observations are desperately required to corroborate their surprising results and make a more definitive investigation.

Given its above history, a new metallicity study of To 2 is imperative. These points motivated the present study, wherein we wished to obtain independent high-resolution spectra with a large telescope of a number of To2 stars in order to investigate the reality of the putative metallicity dispersion and the nature of its chemical composition. In Sect. 2 we describe our observations and reduction procedures. In Sect. 3 we discuss the determination of radial velocities and membership for our observed sample. In Sect. 4 we present the details of our abundance analysis, including the derivation of the input atmospheric parameters and of the internal errors. In Sect. 5 our basic abundance results are presented, and in Sect. 6 we compare these to previous investigations, especially that of F08 in view of the above. Finally, we summarize and emphasize the importance of our main results in Sect. 7.

\section{Observations and data reduction}

Our data-set consists of high resolution spectra collected with FLAMES-GIRAFFE/VLT@UT2 (Pasquini et al. 2002) in Service mode from March 6 to March 25 2006, within a project devoted to measure radial velocities, membership, and chemistry in a large sample of open clusters (Randich et al. 2005). The GIRAFFE spectrograph was used in the HR15N setting, providing a resolution of $R \sim 17000$ and covering a spectral range of $\sim 320 \AA$ with the central wavelength at $6650 \AA$. Typical seeing during the observations was in the range of $0.8-1.2$ arcsec.

The cluster was observed with two different configurations $(\mathrm{A}$ and $\mathrm{B})$, centered at the same position $(\mathrm{RA}(2000)=$ 07h 03m 01.95s, Dec $=-20 \mathrm{~d} 49 \mathrm{~m} \mathrm{50.2s).} \mathrm{We} \mathrm{obtained} \mathrm{four}$ and three $45 \mathrm{~min}$ long exposures for the configurations $\mathrm{A}$ and $\mathrm{B}$, respectively. Medusa fibers were allocated to 93 and 120 stars in the two configurations, with 78 stars in common. Hence, we obtained in total spectra of 135 cluster candidates. These cover the magnitude range of $14 \leq \mathrm{V} \leq 18.7$ and are located in different regions of the cluster color-magnitude diagram (CMD): namely, the turn-off and subgiant branch, the red giant branch (RGB), the red clump (RC), and the blue plume. In this paper we focus on RGB and RC stars for a total of 37 objects. 15 of them turned out to be members (see below for the membership determination), but only 13 had spectra with sufficient quality in order to measure chemical abundances and are listed in Table 1. Their position in the cluster CMDs is shown in Fig. 1. Targets were originally selected from the Phelps et al. (1994) photometry. But in this paper we use our new optical photometry (see following sub-section) and 2MASS (JHK - Skrutskie et al. 2006).

Data were reduced using GIRAFFE pipelines (Ballester et al. 2000), including bias subtraction, flat-field correction, and wavelength calibration. Sky subtraction was performed plate by plate using the median sky as obtained from the fibers pointed on empty regions of the field. Radial velocities were derived from each single spectrum (see below). Spectra of stars that were not found to be $R V$ variables were then co-added. The typical signal to noise ratio per pixel is $S / N \sim 60-80$.

\subsection{Photometric material}

We complemented the spectroscopic observations with new $U B V I_{\mathrm{C}}$ observations secured with the Cerro Tololo InterAmerican Observatory $1.0 \mathrm{~m}$ telescope, operated by the SMARTS consortium as a part of a large photometric program to study stellar fields in the third Galactic Quadrant. Tombaugh 2 was observed in December 2008 under photometric conditions. Full details of the observations, data reduction and photometric calibration can be found in (Carraro \& Costa 2009) and references therein. Here we would just like to point out the large field ( $20^{\prime}$ on a side) and good scale $\left(0.297^{\prime \prime} /\right.$ pixel $)$ provided by our set-up, which allows for an optimum study of both the central cluster and the surrounding field. For the purpose of this study, we selected stars within 3 arcmin from the nominal cluster center and cross-correlated our data with the 2MASS catalog. Typical deep exposures times in $U, B, V$ and $I_{\mathrm{C}}$ were $2000,1500,1200$, and $1200 \mathrm{~s}$, respectively. 
S. Villanova et al.: The metallicity of the open cluster Tombaugh 2
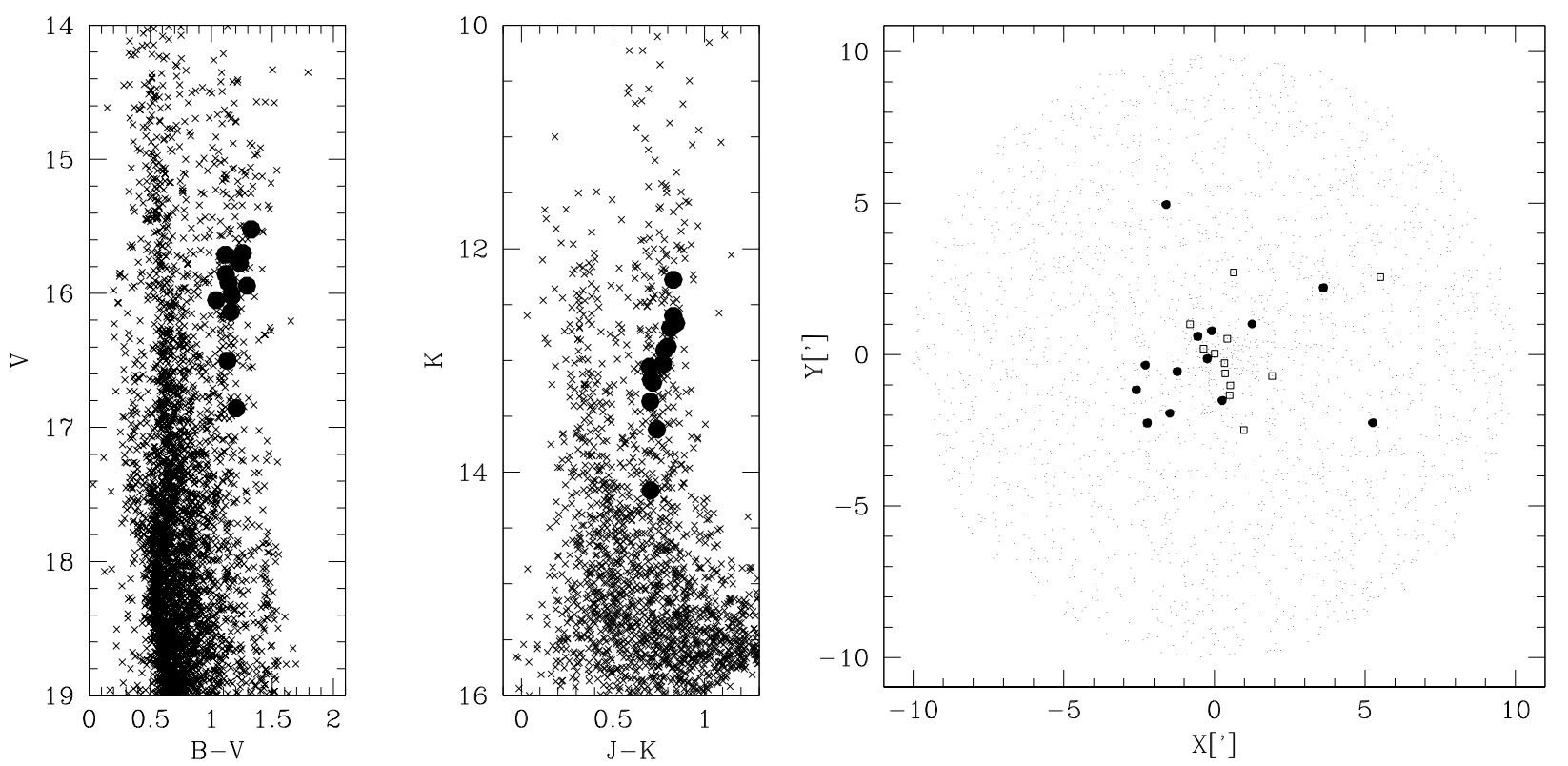

Fig. 1. Left panel: the CMD of Tombaugh 2 in the optical and infrared. Members stars studied in this paper are indicated as filled circles. Right panel: distribution on the sky of our targets (filled points) and F08 objects (empty squares).

Table 1. Target stars and parameters.

\begin{tabular}{|c|c|c|c|c|c|c|c|c|c|c|c|c|c|c|}
\hline ID1 & ID2 & $\alpha\left({ }^{0}\right)$ & $\delta\left({ }^{0}\right)$ & V & $U-B$ & $B-V$ & $V-I_{\mathrm{C}}$ & $J_{2}$ & $H_{2 \text { MASS }}$ & $K_{\text {2MASS }}$ & $T_{\text {eff }}$ & $\log g$ & $v_{\mathrm{t}}$ & $R V_{\mathrm{H}}$ \\
\hline 1512 & 2138 & 105.744536 & -20.848913 & 16.857 & 1.122 & 1.206 & 1.294 & 14.870 & 14.278 & 14.164 & 5210 & 3.29 & 1.10 & 117.6 \\
\hline 1672 & 1100 & 105.724763 & -20.836042 & 15.726 & 0.818 & 1.231 & 1.319 & 13.511 & 12.850 & 12.666 & 4820 & 2.63 & 1.26 & 119.1 \\
\hline 1827 & 837 & 105.748951 & -20.825960 & 15.773 & 0.877 & 1.233 & 1.347 & 13.521 & 12.865 & 12.708 & 4900 & 2.70 & 1.24 & 120.9 \\
\hline 1886 & 1532 & 105.730038 & -20.822460 & 15.711 & 0.638 & 1.116 & 1.251 & 13.675 & 13.024 & 12.876 & 5010 & 2.72 & 1.24 & 120.0 \\
\hline 2184 & 485 & 105.793143 & -20.799812 & 15.857 & 0.702 & 1.118 & 1.262 & 13.755 & 13.214 & 13.055 & 4980 & 2.77 & 1.23 & 119.8 \\
\hline 236 & 2895 & 105.864625 & -20.854195 & 15.919 & 0.669 & 1.141 & 1.281 & 13.804 & 13.234 & 13.031 & 5050 & 2.83 & 1.21 & 121.0 \\
\hline 238 & 1802 & 105.731226 & -20.854284 & 15.699 & 0.889 & 1.258 & 1.341 & 13.431 & 12.771 & 12.601 & 4780 & 2.60 & 1.27 & 120.5 \\
\hline 2846 & 2430 & 105.742321 & -20.734119 & 16.017 & 0.547 & 1.166 & 1.187 & 14.072 & 13.526 & 13.368 & 5160 & 2.92 & 1.19 & 122.9 \\
\hline 299 & 2902 & 105.775540 & -20.841908 & 15.523 & 1.002 & 1.327 & 1.426 & 13.107 & 12.397 & 12.277 & 4780 & 2.53 & 1.29 & 121.8 \\
\hline 3574 & 2074 & 105.766791 & -20.818871 & 16.138 & 0.678 & 1.161 & 1.321 & 13.919 & 13.311 & 13.200 & 5150 & 2.96 & 1.18 & 121.7 \\
\hline 3763 & 2894 & 105.761083 & -20.806566 & 16.051 & 0.783 & 1.041 & 1.288 & 13.883 & 13.367 & 13.175 & 5000 & 2.86 & 1.20 & 120.1 \\
\hline 3836 & 494 & 105.769301 & -20.803593 & 16.501 & 0.704 & 1.133 & 1.382 & 14.357 & 13.750 & 13.617 & 5110 & 3.09 & 1.15 & 120.9 \\
\hline 591 & 2442 & 105.835422 & -20.779896 & 15.944 & 0.706 & 1.293 & 1.355 & 13.690 & 13.018 & 12.909 & 5020 & 2.82 & 1.21 & 125.1 \\
\hline
\end{tabular}

\section{Radial velocities and membership}

In the present work, radial velocities, coupled with the position of the star along the principal giant sequences in the cluster color-magnitude diagram, were used as the membership criterion since cluster stars all have similar motion with respect to the observer. The radial velocities of the stars were measured using the IRAF FXCOR task, which cross-correlates the object spectrum with a template. As a template, we used a synthetic spectrum obtained through the spectral synthesis code SPECTRUM ${ }^{1}$, using a Kurucz model atmosphere (Kurucz 1992) with roughly the mean atmospheric parameters of our stars $T_{\text {eff }}=5000 \mathrm{~K}$, $\log g=2.9, v_{\mathrm{t}}=1.2 \mathrm{~km} \mathrm{~s}^{-1},[\mathrm{Fe} / \mathrm{H}]=-0.30$. At the end, each radial velocity was corrected to the heliocentric system. We calculated a first approximation mean velocity and the rms $(\sigma)$ of the velocity distribution. Stars showing $v_{\mathrm{r}}$ more than $3 \sigma$ from the mean value were considered probable field objects and rejected, leaving us with 15 objects as probable members. Only 13 of them had spectra with sufficient quality in order to measure chemical abundances, and their positions in the CMD and on the

${ }^{1}$ See http://www.phys . appstate. edu/spectrum/spectrum. html for more details. sky are shown in Fig. 1. Radial velocities of these 13 targets are reported in Table 1, while coordinates, $V$ magnitudes and velocities for non-members and the two member with bad spectra are reported in Table 5. We found for the cluster a mean heliocentric radial velocity and an observed dispersion of:

$\left\langle v_{\mathrm{r}}\right\rangle=120.9 \pm 0.4, \sigma_{v_{\mathrm{r}}}=1.7 \pm 0.3 \mathrm{~km} \mathrm{~s}^{-1}$.

Both of these values agree excellently with F08.

Our analysis shows that only 15 out of the 37 sample stars are cluster members, thus yielding a contamination of $\sim 60 \%$.

\section{Abundance analysis}

\subsection{The linelist}

The linelist for the elements we measured (with the exception of $\mathrm{Li}, \mathrm{Ba}$, and $\mathrm{Eu}$ ) were obtained from the VALD database (Kupka et al. 1999) and calibrated using the solar-inverse technique. For this purpose we used the high resolution, high $S / N$ Solar spectrum obtained at NOAO (National Optical Astronomy Observatory, Kurucz et al. 1984). The EWs for the reference solar spectrum were obtained in the same way as the observed spectra (see next subsection) with the exception of the strongest 

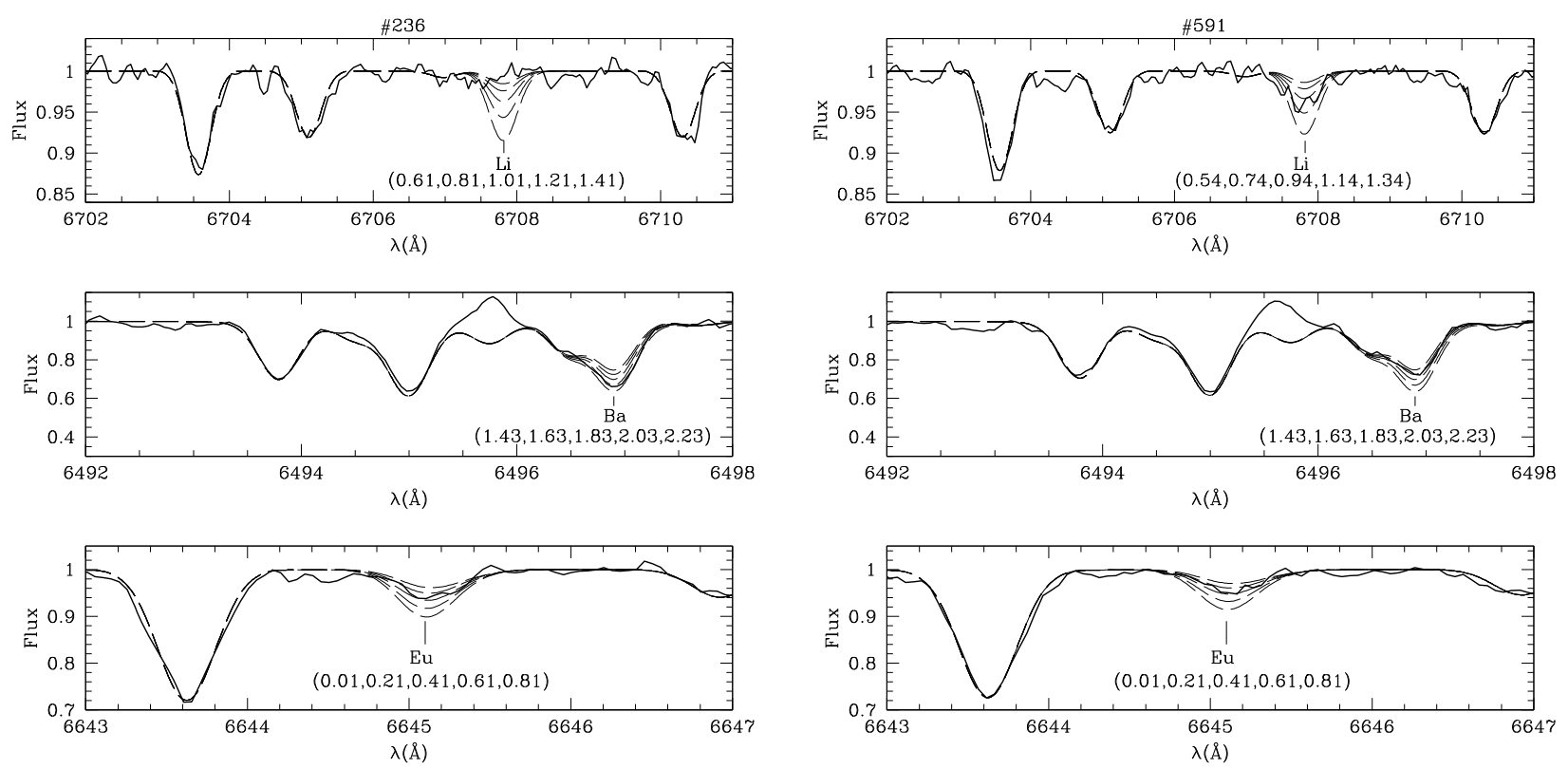

Fig. 2. Example of the spectral synthesis method applied to stars \#236 and \#591. Observed spectra are plotted with continuous lines, while the synthetic ones are plotted with dashed lines. Abundances $\left.\left(\log \epsilon(\mathrm{X})=\log \left(N_{\mathrm{X}} / N_{\mathrm{H}}\right)+12\right)\right)$ used in the synthesis are indicated.

lines, where a Voigt profile integration was used. Lines affected by blends were rejected from the final line-list. Abundances were determined using $\mathrm{MOOG}^{2}$, coupled with a solar model atmosphere interpolated from the Kurucz (1992) grid using the canonical atmospheric parameters for the Sun: $T_{\text {eff }}=5777 \mathrm{~K}$, $\log g=4.44, v_{\mathrm{t}}=0.80 \mathrm{~km} \mathrm{~s}^{-1}$ and $[\mathrm{Fe} / \mathrm{H}]=0.00$. In the calibration procedure, we adjusted the value of the line strength $\log (g f)$ of each spectral line so that the abundances obtained from all the lines of the same element yield the same value. Note that this procedure is identical to that used in F08.

For $\mathrm{Ba}$, known to be affected by hyperfine structure and isotopic effects, we applied the spectrum synthesis method instead. For the Sun we considered Ba lines at 4554, 5853, 6141, and $6496 \AA$. Hyperfine components of those lines and isotopic composition were taken from McWilliam (1998). For each line we determined a $\mathrm{Ba}$ abundance, and then we adjusted the $\log (g f)$ value of each one in order to yield the same abundance obtained from all the lines. For the present data we used only the Ba feature at $6496 \AA$.

The Eu line we used is located at $6645 \AA$ and it is blended with a Si feature. We verified that at the temperature of our stars the contribution of the Si line is negligible. Anyway we obtain Eu abundance by spectral synthesis method. For Si abundance, lacking a direct determination, we assumed the $\alpha$-enhancement as obtained by $\mathrm{Ca}$. The Eu line is so weak that it does not require a hyperfine structure treatment.

The Li line at $6707 \AA$ is an unresolved doublet, so we performed a spectrum synthesis analysis for this element as well.

For Eu and Li we took parameters for the $6707 \AA$ line from VALD and NIST $^{3}$ databases, and the $\log (g f)$ values were simply averaged.

Chemical abundances obtained for the Sun are reported in Table 2 and compared with Grevesse \& Sauval (1998). For Li we report the meteoritic value. We use our abundances instead of those from Grevesse \& Sauval (1998) or Asplund et al. (2005)

\footnotetext{
${ }^{2}$ See http://verdi.as.utexas.edu/moog.html for more details

${ }_{3}$ NIST database can be found at http://physics.nist.gov/ PhysRefData/ASD/lines_form.html
}

Table 2. Our measured solar abundances $\left(\log \epsilon(\mathrm{X})=\log \left(N_{\mathrm{X}} / N_{\mathrm{H}}\right)+12\right)$ compared with Grevesse \& Sauval (1998).

\begin{tabular}{lcc}
\hline \hline Element & $\log \epsilon(\mathrm{X})$ & $\mathrm{GS} 98$ \\
\hline LiI & - & 3.31 \\
$\mathrm{CaI}$ & 6.39 & 6.36 \\
$\mathrm{ScI}$ & 3.12 & 3.17 \\
$\mathrm{FeI}$ & 7.50 & 7.50 \\
$\mathrm{NiI}$ & 6.28 & 6.25 \\
$\mathrm{BaI}$ & 2.34 & 2.13 \\
$\mathrm{Eu}$ & 0.51 & 0.52 \\
\hline
\end{tabular}

Notes. For Li we use the meteoritic value.

because they were obtained in an homogenous way with respect to our target stars.

\subsection{Continuum, atmospheric parameters and chemical abundance determination}

The chemical abundances for $\mathrm{Li}, \mathrm{Ba}$, and $\mathrm{Eu}$ were obtained by comparing observed spectra with synthetic ones (see Fig. 2 for an example of the spectral synthesis applied to stars \#236 and \#591), so in this case the continuum detrermination is a by-product of this procedure. For the remaining elements abundances were obtained from the equivalent widths (EWs) of the spectral lines. In this second case a continuum determination is a bit more complicated. Our spectra are centered on the $\mathrm{H}_{\alpha}$ region, so they are relatively free from spectral lines, especially compared to the data of F08, whose spectral range was much bluer and contains many more lines. This fact allowed us to proceed in the following way. First, we selected for each line a region of $20 \AA$ centered on the line itself (this value is a good compromise between having enough points, i.e. good statistics, and avoiding an excessively large spectral region over which the spectrum could be substantially curved). Then we built the histogram of the distribution of the flux where the peak is a rough estimation of the continuum. We refined this determination by fitting a parabolic curve to the peak and using the vertex as our 
S. Villanova et al.: The metallicity of the open cluster Tombaugh 2

Table 3. Evolutionary stage and chemical abundances $\left(\log \epsilon(\mathrm{X})=\log \left(N_{\mathrm{X}} / N_{\mathrm{H}}\right)+12\right)$ of our objects.

\begin{tabular}{|c|c|c|c|c|c|c|c|c|c|}
\hline ID & Ev. Stage & $\mathrm{FeI}$ & {$[\mathrm{FeI} / \mathrm{H}]$} & LiI & $\mathrm{CaI}$ & ScI & $\mathrm{NiI}$ & BaII & EuII \\
\hline 1512 & RGB & 7.16 & -0.34 & - & 6.11 & 2.70 & 6.05 & 1.61 & - \\
\hline 1672 & RGB & 7.12 & -0.38 & 1.02 & 6.19 & 2.73 & 5.82 & 2.01 & 0.45 \\
\hline 1827 & RGB & 7.25 & -0.25 & 1.25 & 6.35 & 2.91 & 6.15 & 2.20 & 0.58 \\
\hline 1886 & $\mathrm{RC}$ & 7.11 & -0.39 & $<0.40$ & 6.01 & 2.77 & 6.00 & 2.09 & 0.34 \\
\hline 2184 & $\mathrm{RC}$ & 7.19 & -0.31 & $<0.50$ & 6.29 & 2.96 & 6.00 & 2.16 & 0.39 \\
\hline 236 & $\mathrm{RC}$ & 7.20 & -0.30 & - & 6.26 & 2.75 & 5.88 & 2.11 & 0.35 \\
\hline 238 & RGB & 7.20 & -0.30 & 0.68 & 6.25 & 2.88 & 6.00 & 2.02 & 0.44 \\
\hline 2846 & $\mathrm{RC}$ & 7.11 & -0.39 & 1.25 & 6.19 & 3.02 & 5.95 & - & 0.82 \\
\hline 299 & RGB & 7.31 & -0.19 & 0.90 & 6.31 & 2.95 & 6.01 & 2.15 & 0.62 \\
\hline 3574 & $\mathrm{RC}$ & 7.27 & -0.23 & $<0.60$ & 6.28 & 2.83 & 6.06 & 2.23 & 0.54 \\
\hline 3763 & $\mathrm{RC}$ & 7.12 & -0.38 & $<0.40$ & 6.25 & 2.72 & 5.91 & 1.99 & 0.28 \\
\hline 3836 & RGB & 7.26 & -0.24 & 1.26 & 6.28 & 2.74 & 5.88 & 2.14 & 0.57 \\
\hline 591 & RGB & 7.13 & -0.37 & 1.04 & 6.16 & 2.65 & 5.82 & 1.61 & 0.34 \\
\hline Cluster & & & {$[\mathrm{Fe} / \mathrm{H}]=-0.31$} & & {$[\mathrm{Ca} / \mathrm{Fe}]=+0.16$} & $\mathrm{Sc} / \mathrm{Fe}]=-0.01$ & {$[\mathrm{Ni} / \mathrm{Fe}]=+0.00$} & {$[\mathrm{Ba} / \mathrm{Fe}]=+0.07$} & {$[\mathrm{Eu} / \mathrm{Fe}]=+0.23$} \\
\hline Obs. lines & & 16 & 16 & 1 & 3 & 1 & 2 & 1 & 1 \\
\hline
\end{tabular}

Notes. RGB indicates red giant branch stars, while RC indicates red clump stars.

continuum estimation. Finally, the continuum determination was revised by eye and corrected by hand if a clear discrepancy with the spectrum was found. Then, using the continuum value previously obtained, we fit a Gaussian curve to each spectral line and obtained the EW from integration. We rejected lines if affected by bad continuum determination, by non-Gaussian shape, if their central wavelength did not agree with that expected from our line-list, if the lines were too broad or too narrow with respect to the mean FWHM, or if it was affected by blending with other spectral features. We verified that the Gaussian shape was a good approximation for our (mostly weak) spectral lines, so generally no Lorentzian correction was applied. The used lines and the measured EWs are reported in Table 6.

Initial estimates of the atmospheric parameters were derived from $B V I_{\mathrm{C}} J H K$ photometry. Effective temperatures $\left(T_{\text {eff }}\right)$ for each star were derived from $T_{\text {eff }}-[\mathrm{Fe} / \mathrm{H}]$-color relations (Alonso et al. 1999; di Benedetto 1998; Ramírez \& Meléndez 2005). Colors were de-reddened assuming a reddening value of 0.3 mag (Frinchaboy et al. 2008). Then $T_{\text {eff }}$ was adjusted in order that abundances derived for individual FeI lines showed no trend with excitation potential.

Surface gravities $\log g$ were obtained from the canonical equation:

$\log \left(g / g_{\odot}\right)=\log \left(M / M_{\odot}\right)+4 \cdot \log \left(T_{\text {eff }} / T_{\odot}\right)-\log \left(L / L_{\odot}\right)$.

For $M / M_{\odot}$ we adopted $1.45 M_{\odot}$ based on our isochrone fitting.

The luminosity $L / L_{\odot}$ was obtained from the absolute magnitude $M_{V}$ using the measured $V$ magnitude, assuming the bolometric correction (BC) from Alonso et al. (1999) and an apparent distance modulus $(m-M)_{V}$ obtained in the following way.

For many of the spectra we could measure the FeII line at $6456 \AA$. While just one FeII line is not enough to determine a reliable gravity for a single star, it can be used to determine the apparent distance modulus (which is the same for all the stars), simply varying it until the mean FeI and FeII abundances of the cluster (calculated assuming the gravity obtained from the previous formula) are the same. All other quantities in the gravity equation $\left(T_{\mathrm{eff}}, M / M_{\odot}, \mathrm{BC}, V\right)$ are known.

We obtained:

$(m-M)_{V}=15.01 \pm 0.12$.

We point out that this value was optimized in order to give $[\mathrm{FeI} / \mathrm{H}]=[\mathrm{FeII} / \mathrm{H}]$, so it can differ from the real apparent distance modulus because the other variables in the gravity equation (mainly the mass) can be affected by systematic errors. This can explain the difference with F08, where the authors find $(m-M)_{0} \sim 14.5$, while we have $(m-M)_{0} \sim 14.30$ assuming $E(B-V)=0.23$ (see Sect. 5). However, the two values are not incompatible within the errors.

Finally, microturbulence velocity $\left(v_{t}\right)$ was obtained from the relation:

$v_{\mathrm{t}}\left(\mathrm{km} \mathrm{s}^{-1}\right)=-0.254 \cdot \log g+1.930$

which was obtained from Marino et al. (2008).

Adopted atmospheric parameters for each star are reported in Table 1. In this table Cols. 1 and 2 give the ID of the star according to Phelps et al. (1994, ID1) and our photometry (ID2). Columns 3 and 4 give the coordinates, Cols. 5-11 the $B, V, I, J$, $H, K$ magnitudes, Col. 12 the temperature (K), Col. 13 the gravity, Col. 14 the microturbulence velocity $\left(\mathrm{km} \mathrm{s}^{-1}\right)$, and Col. 15 the heliocentric radial velocity $\left(\mathrm{km} \mathrm{s}^{-1}\right)$.

The Local Thermodynamic Equilibrium (LTE) program MOOG was used to determine the abundances, coupled with a model atmosphere interpolated from the Kurucz models for the parameters obtained as described before. Results are reported in Table 3.

\subsection{Internal errors associated with the calculation of the chemical abundances}

The abundances of every element are affected by measurement errors. In this section our goal is to estimate the total amount of the internal error $\left(\sigma_{\text {tot }}\right)$ affecting our data. Clearly, this requires an accurate analysis of all the internal sources of errors. External errors can be estimated by comparison with other works, as done in Sect. 6.

It must be noted that two sources of error mainly contribute to $\sigma_{\text {tot }}$. They are:

- the errors $\sigma_{\mathrm{EW}}$ due to the uncertainties in the EWs measures or in the comparison of observed spectra with synthetic ones;

- the uncertainty $\sigma_{\text {atm }}$ introduced by errors in the atmospheric parameters adopted to compute the chemical abundances.

$\sigma_{\text {EW }}$ is given by MOOG for each element and each star in the case of $\mathrm{Ca}, \mathrm{Fe}$, and $\mathrm{Ni}$. For $\mathrm{Li}, \mathrm{Ba}$, and $\mathrm{Eu}$, whose abundances 
Table 4. Internal errors associated to the chemical abundances.

\begin{tabular}{lcccccc}
\hline \hline El. & $\sigma_{\text {EW }}$ & $\Delta T_{\text {eff }}=50 \mathrm{~K}$ & $\Delta \log g=0.06$ & $\Delta v_{\mathrm{t}}=0.02 \mathrm{~km} \mathrm{~s}^{-1}$ & $\sigma_{\text {tot }}$ & $\sigma_{\text {obs }}$ \\
\hline$\Delta[\mathrm{FeI} / \mathrm{H}]$ & 0.03 & 0.06 & 0.02 & 0.02 & 0.07 & 0.07 \\
$\Delta \log \epsilon(\mathrm{Li})$ & 0.10 & 0.01 & 0.00 & 0.01 & 0.10 & $>0.2$ \\
$\Delta[\mathrm{CaI} / \mathrm{FeI}]$ & 0.06 & 0.01 & 0.00 & 0.01 & 0.06 & 0.05 \\
$\Delta[\mathrm{ScI} / \mathrm{FeI}]$ & 0.10 & 0.01 & 0.00 & 0.01 & 0.10 & 0.08 \\
$\Delta[\mathrm{NiI} / \mathrm{FeI}]$ & 0.07 & 0.02 & 0.01 & 0.02 & 0.08 & 0.09 \\
$\Delta[\mathrm{BaII} / \mathrm{FeI}]$ & 0.06 & 0.01 & 0.03 & 0.00 & 0.07 & 0.06 \\
$\Delta[\mathrm{EuII} / \mathrm{FeI}]$ & 0.05 & 0.02 & 0.02 & 0.00 & 0.06 & 0.07 \\
\hline
\end{tabular}

were obtained by the spectral synthesis method. $\sigma_{\text {EW }}$ was obtained using the $\chi^{2}$ method, as described in Villanova et al. (2009). In Table 4 we report in the second column the average $\sigma_{\mathrm{EW}}$ for each element. For Sc we were able to measure only one line. For this reason its $\sigma_{\mathrm{EW}}$ has been obtained as the mean of $\sigma_{\text {EW }}$ of Ni multiplied by $\sqrt{2}$.

Errors in temperature were determined as in Marino et al. (2008). The mean error $\Delta T_{\text {eff }}$ turned out to be $\sim 50 \mathrm{~K}$. The uncertainty in the surface gravity has been obtained by the canonical formula using the propagation of errors. The variables used in this formula that are affected by random errors are $T_{\text {eff }}$ and the $V$ magnitude. For the temperature we used the error previously obtained, while for $V$ we assumed a mean error of 0.1 mag, which is the typical random error for stars at that magnitude. Other error sources (distance modulus, reddening, bolometric correction, mass) affect gravity in a systematic way, so are not important to our analysis. The mean gravity error turned out to be 0.06 dex. This implies a mean error in the microturbulence of $0.02 \mathrm{~km} \mathrm{~s}^{-1}$.

Once the internal errors associated with the atmospheric parameters were calculated, we re-derived the abundances of one reference star (\#591), assumed to represent our sample, by using the following combination of atmospheric parameters:

$$
\begin{aligned}
& -\left(T_{\text {eff }} \pm \Delta\left(T_{\text {eff }}\right), \log g, v_{\mathrm{t}}\right) \\
& -\left(T_{\text {eff }}, \log g \pm \Delta(\log g), v_{\mathrm{t}}\right) \\
& -\left(T_{\text {eff }}, \log g, v_{\mathrm{t}} \pm \Delta\left(v_{\mathrm{t}}\right)\right)
\end{aligned}
$$

where $T_{\text {eff }}, \log g, v_{\mathrm{t}}$ are the measures determined in this section.

The difference of abundance between values obtained with the original and modified values gives the errors in the chemical abundances due to uncertainties in each atmospheric parameter. They are listed in Table 4 (Cols. 3-5). Our best estimate of the total error associated to the abundance measures is calculated as

$\sigma_{\text {tot }}=\sqrt{\sigma_{\mathrm{EW}}^{2}+\Sigma\left(\sigma_{\mathrm{atm}}^{2}\right)}$

listed in the Col. 6 of Table 4. Column 7 of Table 4 is the observed dispersion obtained as described in Sect. 5. Comparing $\sigma_{\text {obs }}$ with $\sigma_{\text {tot }}$ we can see that almost all the elements ( $\mathrm{Ca}, \mathrm{Sc}, \mathrm{Fe}, \mathrm{Ni}, \mathrm{Ba}$, and $\mathrm{Eu}$ ) have an abundance that is homogeneous with no evidence of spread. The two Ba-poor stars (\#1512 and \#591) and the Li trend will be discussed in the following section.

\section{Results of abundance analysis and revised cluster parameters}

The chemical abundances we obtained are summarized in Table 3 together with the evolutionary stage of each star. This latter is based on the star position in the CMD (Fig. 5), where there is a clear division between RGB and RC stars. The metallicity of the cluster turns out to be sub-solar with:

$[\mathrm{Fe} / \mathrm{H}]=-0.31 \pm 0.02 \mathrm{dex}$.

The abundance ratio trends versus $[\mathrm{Fe} / \mathrm{H}]$ with the exception of $\mathrm{Li}$ are shown in Fig. 3. The bottom right panel report also the $[\mathrm{Eu} / \mathrm{Ba}]$ content, which is an indicator of the r-process/s-process element ratio, because $\mathrm{Eu}$ is an almost pure r-element, while $\mathrm{Ba}$ is mainly produced in s-processes. Mean abundance ratios were calculated using the sigma clipping rejection method. Rejected measures are plotted as open circles. Mean values are reported in Fig. 3 and Table 3. The cluster turns out to have a solar scaled composition for $\mathrm{Sc}$ and $\mathrm{Ni}$, while $\mathrm{Ba}$ is slightly overabundant and Eu is overabundant. $\mathrm{Ca}$ is overabundant, and its value $(+0.16)$ is typical of a cluster of this metallicity. The $\mathrm{Ca}$ overabundance found here is consistent with the Ti overabundance found in F08, as expected from these $\alpha$-elements. It is interesting to note that two stars (\#1512 and \#591, see Fig. 2) have a very low Ba content ( $\sim-0.5$ with respect to the other stars) that cannot be explained with measurement errors, which are of the order of 0.1 dex. The explanation could be an evolutionary effect, but these two stars are located in the same region of the CMD with respect to the other targets, so this hypothesis is unlikely. The only remaining reason that occurs to us is some problem in the data (bad flat-fielding?), but no evidence for this was found in a further check. In any case \#1512 and \#591 were rejected as suspect objects as far as Ba content is concerned.

The mean $[\mathrm{Eu} / \mathrm{Ba}]$ value turns out to be $+0.17 \mathrm{dex}$. This is an indication that Tombaugh 2 could be a intermediate object between the thin and thick disk, according to Mashonkina et al. (2003, see their Fig. 4). In fact in that paper thick disk stars have $[\mathrm{Eu} / \mathrm{Ba}]$ greater than $0.35 \mathrm{dex}$, while thin disk stars have $[\mathrm{Eu} / \mathrm{Ba}] \sim 0.0$ on average. However, its large distance from the Galactic plane $(\sim 700 \mathrm{pc})$ makes it a certain thick disk member. According to the measured $[\mathrm{Eu} / \mathrm{Ba}]$ value, stars of Tombaugh 2 were formed from material more enriched by r-process elements with respect to the Sun, being the cluster $\sim 1.5$ times richer in Eu that our star.

$\mathrm{Li}$ is a very fragile element, which is easily destroyed in stellar interiors at relatively low temperature $\left(2.5 \times 10^{6} \mathrm{~K}\right)$. During the life of a star and in particular during the MS phases, the Li-rich material that lies near the surface is circulated downwards where the temperature is high enough for Li burning to occur. When the star evolves to the red giant phase, the deepening of the convective envelope brings up to the surface internal matter which was nuclearly processed and the $\mathrm{Li}$ abundance decreases. Several studies have shown that further Li depletion occurs after the first dredge-up, evidencing the action of an extramixing process (e.g. Charbonnel 1995). This can be seen in Fig. 4, where we plot $\log \epsilon(\mathrm{Li})$ vs. temperature. RGB stars are indicated as filled circles, while RC stars are open squares. One immediately notes that RGB objects have a mean Li abundance 
S. Villanova et al.: The metallicity of the open cluster Tombaugh 2
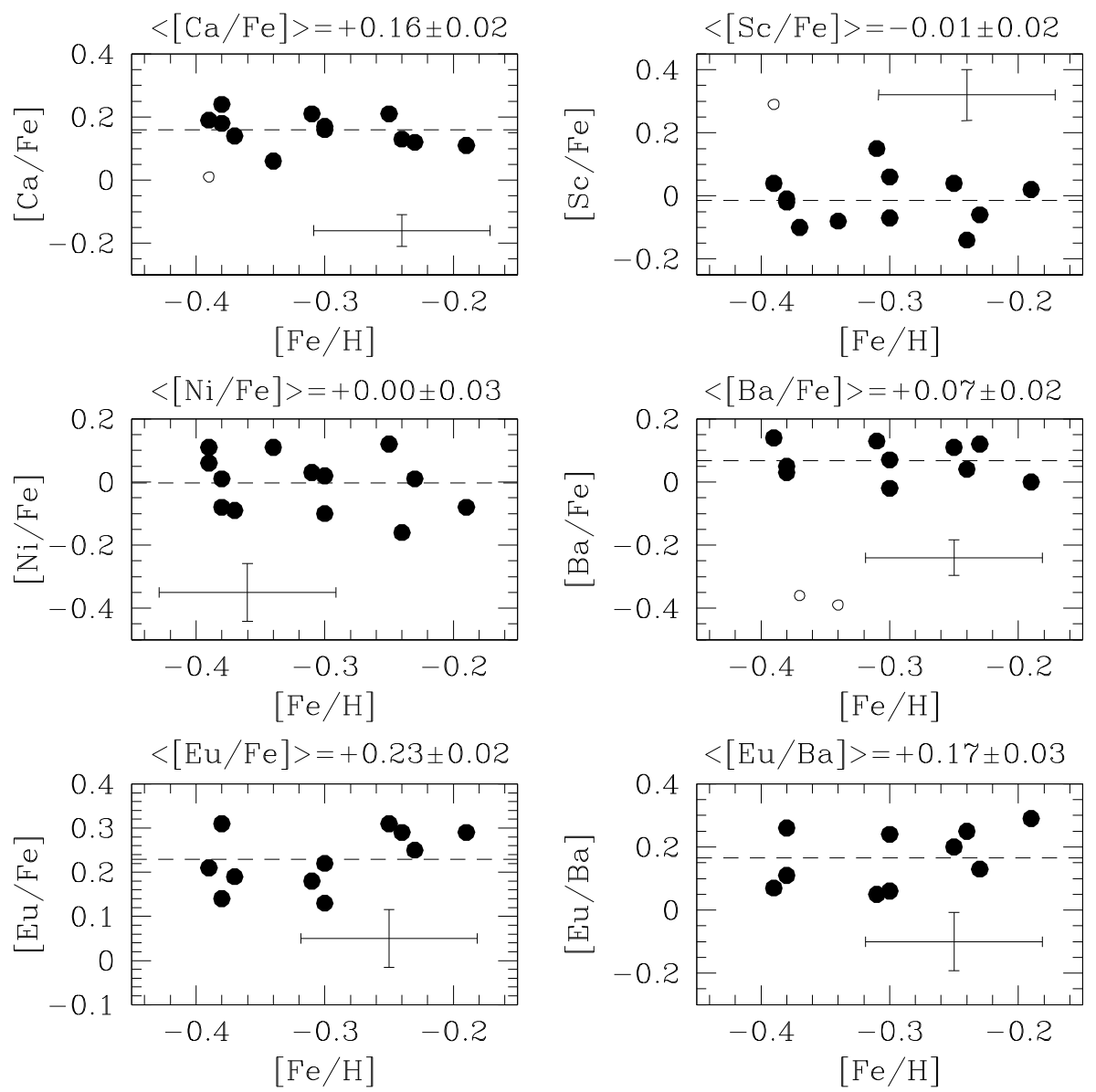

Fig. 3. Abundance ratios obtained for our stars. Typical error bars and mean values (dashed lines) are indicated. Open circles are values rejected during the sigma clipping rejection procedure (see Sect. 5).

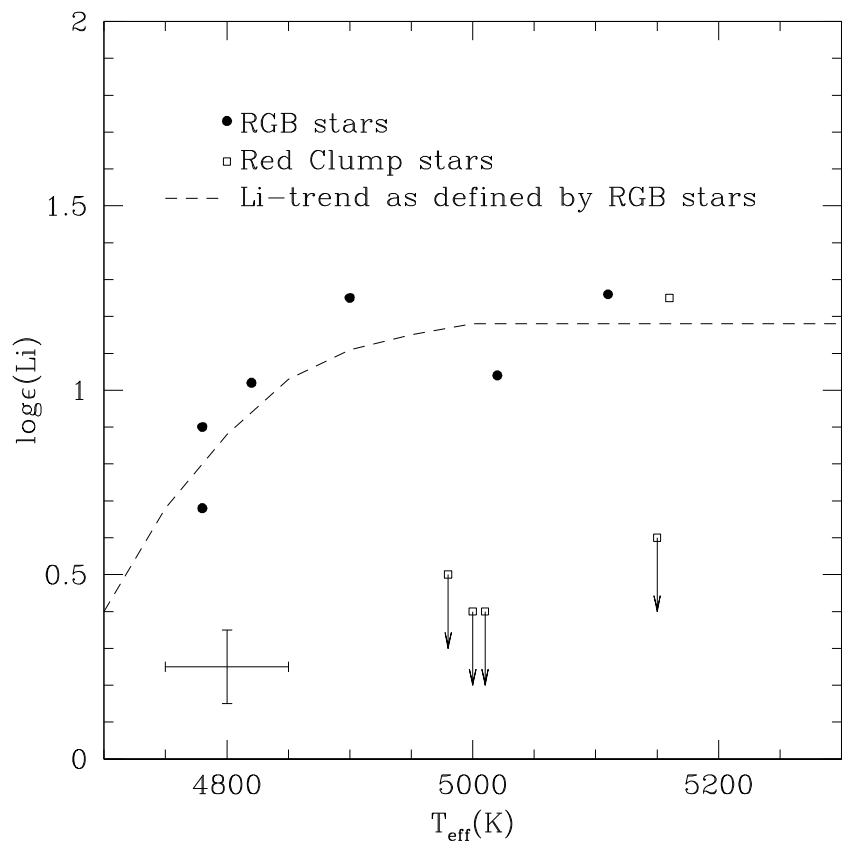

Fig. 4. Li abundances for observed stars. The evolutionary stage of each star is indicated. The symbols with arrows represent upper limits. Typical error bars are indicated.

greater than RC stars, for which we could measure only upper limits (see i.e. stars \#236 and \#591 in Fig. 2). Only the star \#2846 falls out of this picture because it is appears to be a RC object, but its Li content is high (as high as the other RGB stars). This object could be explained by error in the photometry, which altered its real position in the CMD. Otherwise, we found a clear trend of $\mathrm{Li}$ as a function of $T_{\text {eff }}$ for RGB stars. Apparently objects hotter than $4900 \mathrm{~K}$ (and located in the lower RGB) have a constant $\mathrm{Li}$ abundance $(\log \epsilon(\mathrm{Li}) \sim 1.18)$. For stars colder than this limit Li starts to be more and more depleted as the stars climb up the RGB (see dashed line in Fig. 4). A very similar behavior was found by De Freitas et al. (in prep.) based on UVES spectra of several open cluster giant members.

Having new values for metallicity and $\alpha$-element content, we redetermined the basic parameters for the cluster using Padova isochrones (Marigo et al. 2008) and our $V, I$ photometry. The apparent distance modulus we found $\left((m-M)_{V}=15.1\right)$ agrees well with the one from the FeI/II ionization equilibrium. According to our fit (see Fig. 5), Tombaugh 2 has a reddening of $E(B-V)=$ $0.25(E(V-I)=0.32)$ and an age of $2.0 \pm 0.1 \mathrm{Gyr}$, which agrees well with the value given by F08.

\section{Comparison with previous metallicity studies}

We first compare our findings with those of Brown et al. (1996) and Friel et al. (2002). The former conducted a high resolution $(R \sim 34000)$ study of To2 with the CTIO 4-m telescope and found $[\mathrm{Fe} / \mathrm{H}]=-0.40 \pm 0.25$ for $E(B-V)=0.4$ or $[\mathrm{Fe} / \mathrm{H}]=-0.5 \pm 0.25$ for $E(B-V)=0.3$ based on three stars, with $[\mathrm{Fe} / \mathrm{H}]=-0.2,-0.4$, and -0.6 . The Brown abundance 


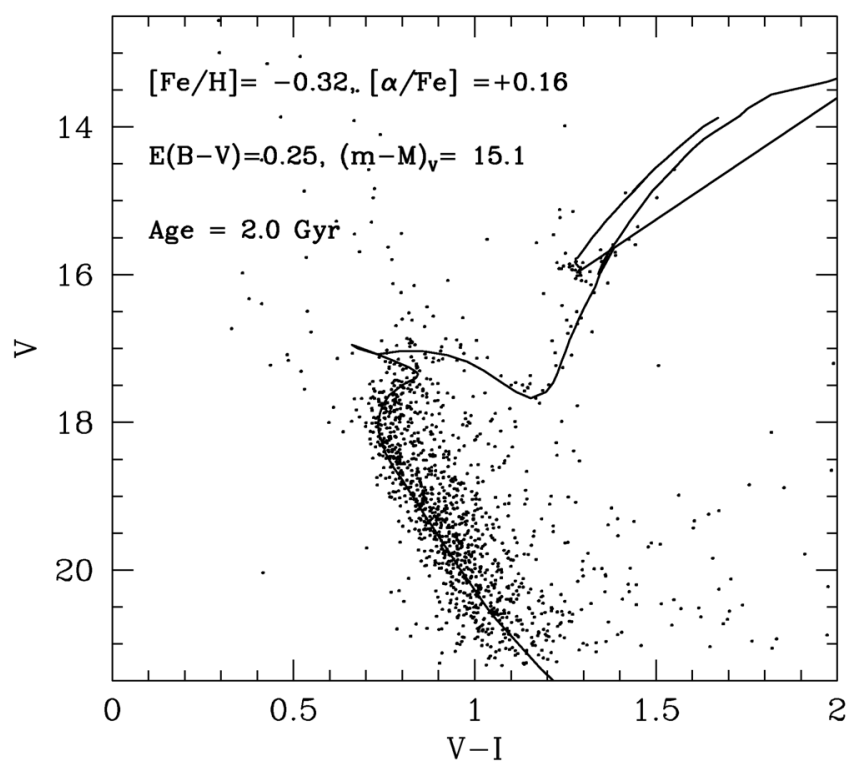

Fig. 5. Isochrone fitting to the CMD. The derived parameters are indicated.

analysis showed that To2 has a reddening of $E(B-V)=0.3-0.4$. The metallicities of their three stars are on the low end of the range of our derived $[\mathrm{Fe} / \mathrm{H}]$ values. However, the more metalrich of the three Brown et al. stars is ruled out as a cluster member based on our radial velocity criterion. Thus, accounting for this, the Brown et al. metallicity would be lower than our mean value, by -0.2 to -0.3 dex, but still within $1 \sigma$ of our value.

Friel et al. (2002) also obtained spectroscopic abundances for To2, using the CTIO 4-m/ARGUS, which yielded a much lower resolution $(R \sim 1300)$ than either our study, F08 or that of Brown et al. (1996), and had metallicities determined from spectral indices. Friel found $[\mathrm{Fe} / \mathrm{H}]=-0.44 \pm 0.09$ for $\mathrm{To} 2$ from a sample of 12 member stars and the individual measurements ranging from -0.28 to -0.65 . Their value agrees well with ours.

We now turn to a comparison with F08. Recall that they also used the VLT, obtaining both high resolution UVES data as well as lower resolution GIRAFFE data. However, their UVES spectra were of very low $S / N, \sim 15-20$, while GIRAFFE data were taken in a much more crowded region of the spectrum 4750-6800 $\AA$ - than we employ here - 6500-6800 $\AA$. Their GIRAFFE resolution $(R=26000)$ is only slightly higher than ours. They derived detailed abundances for 18 cluster members (4 from UVES data), i.e. with photometry consistent with being RGB or RC stars and with velocities in the range $121 \pm$ $4 \mathrm{~km} \mathrm{~s}^{-1}$. They found evidence for two populations of To 2 stars, with a metal-poor group (mean $[\mathrm{Fe} / \mathrm{H}]=-0.28$ ) and a metalrich group (mean $[\mathrm{Fe} / \mathrm{H}]=-0.06$ ), with a fairly small dispersion among each group. We refer to these as the MP and MR groups subsequently. The total metallicity range covered a wide margin: 0.00 to -0.43 . Their data also suggested distinct $\mathrm{Ti}$ abundances for these 2 groups, with the MP group being $\alpha$-enhanced $([\mathrm{Ti} / \mathrm{Fe}]=+0.36)$ and the MR group solar $([\mathrm{Ti} / \mathrm{Fe}]=+0.02)$. In addition, they found that the MP group was more centrally concentrated than their MR counterparts. They argued that their errors were unable to account for these very surprising results and suggested that they were indeed real. After discussing a number of possible scenarios to explain their findings, F08 argued that the most likely was that the centrally concentrated MP group represented the true To2 cluster stars and that the MR group was a spatially overlapping and kinematically
Table 5. The 22 non-member stars and the two members with bad spectra.

\begin{tabular}{lcccc}
\hline \hline ID1 & $\alpha\left(^{0}\right)$ & $\delta\left(^{0}\right)$ & $V$ & $R V_{\mathrm{H}}$ \\
\hline 1160 & 105.807125 & -20.882889 & 15.735 & 74.5 \\
153 & 105.771708 & -20.879250 & 16.990 & 61.1 \\
1530 & 105.674958 & -20.847750 & 15.448 & 98.9 \\
1573 & 105.888125 & -20.841778 & 15.781 & 59.8 \\
169 & 105.814208 & -20.873833 & 15.486 & 90.0 \\
1755 & 105.748833 & -20.830417 & 14.790 & 126.9 \\
2185 & 105.722083 & -20.800333 & 16.286 & 96.3 \\
2343 & 105.712958 & -20.787528 & 15.966 & 104.0 \\
2403 & 105.892917 & -20.781333 & 15.596 & 64.8 \\
250 & 105.789333 & -20.850444 & 17.132 & 87.2 \\
2510 & 105.861958 & -20.771361 & 17.359 & 18.3 \\
266 & 105.885417 & -20.846667 & 15.820 & 130.9 \\
2822 & 105.703542 & -20.736306 & 16.412 & 133.5 \\
2827 & 105.836417 & -20.735028 & 17.007 & 38.5 \\
2911 & 105.678292 & -20.725833 & 15.485 & 109.1 \\
2975 & 105.742917 & -20.717972 & 15.789 & 105.6 \\
3332 & 105.751292 & -20.778472 & 16.991 & 130.6 \\
3562 & 105.768583 & -20.821722 & 15.877 & 51.3 \\
390 & 105.755542 & -20.824139 & 16.032 & 114.9 \\
472 & 105.853083 & -20.803694 & 15.367 & 53.6 \\
589 & 105.847417 & -20.780333 & 16.109 & 135.0 \\
650 & 105.891542 & -20.766750 & 15.736 & 107.2 \\
\hline 4708 & 105.808750 & -20.879833 & 16.899 & 121.8 \\
651 & 105.675708 & -20.768111 & 15.921 & 121.7 \\
\hline & & & &
\end{tabular}

associated but cold stellar stream. They found this scenario to be more feasible than the even more dubious possibility that To2 possessed a real metallicity spread, which would make it unique among Milky Way open clusters, but also claimed this as a viable alternative. Finally, they associated To2 as a likely member of the GASS/Mon stream.

Our results yield a mean metallicity of $-0.31 \pm 0.02$ from 13 members, with values varying from -0.19 to -0.39 , all lying in the range of F08's MP group. This variation is less than half of that covered by all of F08 stars, already a strong hint that F08 may have underestimated their errors. Of equal importance, we find no hint of bimodality in the metallicity distribution. Our errors, which we estimate to be of the same order as those estimated by F08, can completely account for our observed Fe abundance variation. What about stars in common between the two studies? There are six, and the detailed comparison is as follows, where we give (our ID: F08 ID, our $[\mathrm{Fe} / \mathrm{H}]$ : F08 $[\mathrm{Fe} / \mathrm{H}])$. (1672:135, -0.38:-0.07), (1827:140, -0.25:0.00), (238:127, -0.30:-0.34), (3574:199,-0.23:-0.20), (3763:182, $-0.38:-0.03)$, and $(591: 164,-0.37:-0.11)$. Of these, all but the latter were both observed with GIRAFFE. Only two of the stars show good agreement, while the other four consistently have lower metallicities in our study than what was found by F08, in particular the lone UVES star. All four of these stars lie in their MR group, but now we find that actually they have metallicities that would have placed them in their MP group. The differences are substantially larger than expected, given the combined error estimates. Note that all four UVES stars, all with very low $S / N$, lie in the MR group.

How do we explain the discrepancy between our results and those of F08? Is it possible to explain it through differences in the stellar parameters of their two groups? We indeed find that their MP stars are on average about $50 \mathrm{~K}$ cooler, have a $\log g$ some 0.3 smaller and a $v_{\mathrm{t}} \sim 0.05$ less than their MR stars. The combined effects of these differences on the derived metal abundance, based on their estimates of this value to the input 
Table 6. The linelist.

\begin{tabular}{|c|c|c|c|c|c|c|c|c|c|c|c|c|c|c|}
\hline El. & $\lambda(\AA)$ & 1512 & 1672 & 1827 & 1886 & 2184 & 236 & 238 & 2846 & 299 & 3574 & 3763 & 3836 & 591 \\
\hline $\mathrm{FeI}$ & 6494.98 & 154.5 & 225.3 & - & 188.8 & 203.3 & 180.1 & 224.5 & - & 243.9 & 180.9 & 191.7 & 186.0 & 185.3 \\
\hline $\mathrm{FeI}$ & 6498.94 & 80.1 & - & - & - & - & 91.2 & 109.3 & - & 122.7 & 92.6 & - & - & - \\
\hline $\mathrm{FeI}$ & 6533.93 & - & 56.9 & 60.3 & 53.8 & 54.2 & - & 61.5 & 46.2 & 75.5 & 55.0 & 49.3 & 54.9 & 46.3 \\
\hline $\mathrm{FeI}$ & 6569.22 & 88.1 & - & 93.2 & - & - & 92.8 & 92.9 & - & 103.4 & 94.8 & 104.3 & 86.8 & 88.9 \\
\hline $\mathrm{FeI}$ & 6593.87 & - & 122.3 & - & 106.0 & - & 104.4 & 129.7 & - & 127.3 & 95.3 & - & - & 108.0 \\
\hline FeI & 6608.03 & 26.6 & 47.4 & 51.0 & 39.1 & 41.4 & 32.3 & 54.4 & - & 59.3 & - & 41.0 & 37.2 & - \\
\hline $\mathrm{FeI}$ & 6627.54 & 24.5 & 40.3 & 38.6 & 32.2 & 35.1 & 26.6 & 47.9 & _- & 44.9 & - & 41.7 & 35.2 & 34.0 \\
\hline $\mathrm{FeI}$ & 6646.93 & 20.2 & 29.0 & 30.7 & 23.4 & - & - & 37.2 & - & 42.2 & - & 28.1 & - & 26.5 \\
\hline $\mathrm{FeI}$ & 6677.99 & 134.6 & 171.4 & 172.3 & 151.5 & 160.4 & 158.7 & 176.2 & 140.3 & 186.0 & 148.0 & 153.7 & 153.3 & 142.9 \\
\hline $\mathrm{FeI}$ & 6713.74 & - & 29.5 & - & - & - & - & - & 26.5 & - & - & - & - & - \\
\hline FeI & 6726.66 & 46.5 & 58.3 & 56.2 & 50.2 & 55.3 & 61.6 & 59.9 & 40.0 & 60.8 & 53.5 & 50.4 & 54.8 & 46.4 \\
\hline FeI & 6733.15 & 27.6 & 35.8 & 38.0 & 30.9 & 36.7 & 30.4 & 36.8 & 27.4 & 38.2 & 32.1 & 30.5 & 29.9 & 31.7 \\
\hline FeI & 6739.52 & 25.8 & 50.5 & 49.7 & - & 40.6 & - & 53.5 & - & 54.9 & - & 34.9 & 39.8 & 32.8 \\
\hline FeI & 6750.15 & 88.8 & 107.2 & 111.2 & 100.5 & 105.3 & 103.1 & 116.6 & 93.4 & 118.3 & 91.6 & 98.4 & 96.0 & 100.4 \\
\hline FeI & 6752.71 & 42.1 & - & 54.3 & 43.2 & 50.6 & 44.4 & - & - & 61.3 & 42.4 & 41.0 & 45.7 & 40.5 \\
\hline FeI & 6806.84 & 30.5 & - & - & 53.9 & 60.9 & 60.9 & 67.7 & 39.6 & 77.7 & 59.6 & 52.9 & 56.8 & 59.8 \\
\hline FeII & 6456.38 & 70.3 & 63.2 & 67.5 & 73.0 & 79.2 & 64.4 & 57.0 & - & 71.9 & 68.5 & 66.9 & - & - \\
\hline $\mathrm{CaI}$ & 6455.60 & - & - & - & - & - & 80.9 & 91.9 & - & 89.5 & - & - & - & - \\
\hline $\mathrm{CaI}$ & 6471.66 & 101.4 & 124.4 & 128.5 & - & 110.7 & 112.2 & 126.4 & 110.3 & 135.1 & 117.6 & 114.9 & 109.7 & 107.4 \\
\hline $\mathrm{CaI}$ & 6499.65 & 82.1 & 105.1 & 112.8 & 92.2 & 112.4 & 93.6 & 111.4 & 83.5 & 122.4 & 91.5 & 101.9 & 101.5 & 96.3 \\
\hline ScII & 6604.60 & 50.4 & 65.9 & 69.1 & 67.6 & 72.4 & 60.9 & 71.3 & 75.1 & 73.7 & 44.5 & 61.9 & 52.6 & 58.6 \\
\hline NiI & 6767.77 & 107.0 & 114.0 & 124.0 & 110.3 & 119.3 & 105.7 & - & 109.5 & 127.2 & 111.1 & 106.5 & 102.5 & 109.0 \\
\hline NiI & 6772.31 & 56.2 & 63.2 & 78.4 & 70.3 & 62.4 & 57.2 & 69.4 & 50.2 & 71.0 & 60.3 & 63.6 & 51.8 & 50.9 \\
\hline
\end{tabular}

Notes. EWs are reported in $\mathrm{m} \AA$.

atmospheric parameters, can only explain about 0.05 dex of the 0.22 dex difference between the two mean metallicities and is thus insufficient. We feel that the most likely explanation is that F08 seriously underestimated their errors. In particular, their UVES spectra are all of a very low quality. On the other hand the spectral regions of GIRAFFE spectra lie substantially blueward of our spectra, in much more crowded regions. All this makes EWs more difficult to be measured and atmospheric parameters consequently less accurate than those obtained in this paper.

Thus, we do not agree with the main result of F08, viz. that a real abundance spread exists among To2 stars and/or stars associated with the purported stellar stream. There is now no need for such a stream - all stars are simply To 2 members, and all have the same metallicity. Our stars cover an even larger area than those of F08 (see Fig. 1), yet there is no sign of any radial metallicity dependence, another result they suggested. It appears that F08 results on their MP group are actually applicable to all To2 members. A further advantage of our metallicity is that it agrees much better with that expected for a cluster at the Galactocentric distance of To2, which in fact was used as one of the arguments by F08 for preferring the MP group as a representative of the cluster. As they point out, stars as metal rich as those in their MR group (nearly solar metallicity) at $R_{\mathrm{gc}} \geq$ $15 \mathrm{kpc}$ are not consistent with the measured metallicities of red giants in the outer disk (Carney et al. 2005) as well as outer disk Cepheid stars (Yong et al. 2006), which both suggest that the median disk metallicity at this Galactocentric distance should be $[\mathrm{Fe} / \mathrm{H}] \sim-0.4$, similar to our To 2 value. These studies of outer disk tracers find no stars as metal rich as the F08 MR group, even for the younger Cepheid populations. These outer disk stars also show enhanced $\alpha$-element abundances. Indeed, if F08 results for their MP stars are correct, then To2 stars are Ti-enhanced $(\langle[\mathrm{Ti} / \mathrm{Fe}]\rangle=+0.36)$, and we also find them to be Ca enhanced $(\langle[\mathrm{Ca} / \mathrm{Fe}]\rangle=+0.16)$, as found by Carney et al. (2005) and Yong et al. (2006) for outer disk stars. However, we do not find any trend of $[\mathrm{Ca} / \mathrm{Fe}]$ vs. $[\mathrm{Fe} / \mathrm{H}]$, as $\mathrm{F} 08$ found for Ti. Again, evidence for a single chemical composition for To2 stars, as we find, appears stronger and much more tractable than any of the F08 alternatives. Finally, we note that the slight Ba overabundance measured by us is in perfect agreement with the results of D'Orazi et al. (2009) who reported the discovery of a trend of increasing barium abundance with decreasing age based on a large sample of Galactic open clusters. Actually, the inclusion of To2 in the sample allows for better sampling of the age interval between 2 and 4 Gyr.

To conclude, we have no additional evidence for or against F08's contention that To 2 is indeed a likely member of the GASS/Mon stream. However, their argument which read that added weight is given to this possibility by their observed To2 metallicity spread, is now rebuked. They argued that, since NGC 2808 is also believed to be a member and it shows evidence for an internal population dispersion (Piotto et al. 2007), the fact that To 2 also shows such a dispersion favors its GASS/Mon membership.

\section{Discussion and summary}

The existence of multiple populations in Galactic globular clusters and some large magellanic cloud clusters is now well established (Piotto 2008; Marino et al. 2008, 2009; Milone et al. 2009). Indeed, $\omega$ Centauri is now known to possess at least five populations showing different ages and abundances (Villanova et al. 2007). However, certainly not all such clusters show this phenomenon, at least not in a clear way. Indeed, the dominant characteristic that seems to be in common among such clusters is their mass - only the most massive clusters are involved. Given the current paradigm concerning the origin of these multiple populations - retention of gas left over and polluted by a first generation of star formation to form a second, chemically distinct population - obviously requires sufficient cluster mass 
to retain the required $\mathrm{SN}$ and $\mathrm{AGB}$ or massive star wind ejecta. The required mass is estimated to be some $10^{5} M_{\odot}$ (Mieske et al. 2008). Thus, only the most massive Galactic globulars fulfill this requirement (although note that at least one less massive cluster, M4 at $10^{4} M_{\odot}$, also shows multiple populations, Marino et al. 2008). The case of the LMC clusters is more uncertain, but it is clear that the phenomenon is correlated with mass. On the other hand, Galactic open clusters generally do not exceed masses of $10^{3}-10^{4} M_{\odot}$, so that the possibility of retaining any first generation ejecta is very unlikely - any gas that remains after the first star formation episode is subsequently quickly removed by the ejecta itself. Thus, one does not expect to find multiple populations in open clusters. To2 is a typical open cluster in this respect and certainly does not exceed a few $\times 10^{3} M_{\odot}$.

The finding by F08 that To 2 possessed an intrinsic metallicity spread (or was a superposition of an outer disk cluster well out of the plane and a cold stellar stream with exactly the same velocity but distinct chemistry) was then understandably met with some incredulity and at the very least required independent corroboration. Hence the present study.

We obtained independent data, but used the same telescope and spectrograph as they did. However, we deliberately achieved superior data quality - in both $S / N$ and by observing in a longer wavelength regime, where line crowding was significantly reduced. Our reduction and analysis procedures were virtually identical to theirs.

To summarize, we obtained high resolution VLT+GIRAFFE spectra in the optical for a number of stars in the Tombaugh 2 field. Radial velocities and the position in the color-magnitude diagram are used to assess cluster membership. The spectra, together with input atmospheric parameters and model atmospheres, are used to determine detailed chemical abundances for a variety of elements in 13 stars confirmed as members.

We derive a mean metallicity $[\mathrm{Fe} / \mathrm{H}]=-0.31 \pm 0.02$, with no evidence for an intrinsic abundance dispersion. We find $\mathrm{Ca}, \mathrm{Ba}$, and $\mathrm{Eu}$ to be enhanced while $\mathrm{Ni}$ and $\mathrm{Sc}$ are solar. Li abundances decrease with $T_{\text {eff }}$ on the upper giant branch and maintain a low level for red clump stars. The mean metallicity we derive agrees well with that expected from the radial abundance gradient in the disk for a cluster at its Galactocentric distance. The surprising result found by F08, viz. two distinct abundance groups within the cluster, implying either a completely unique open cluster with an intrinsic metallicity spread, or a very unlikely superposition of a cold stellar stream and a very distant open cluster, is not supported by our new data. We suspect that the F08 data was subject to substantially larger errors than they estimated, especially given the low $S / N$ of their UVES spectra and their much bluer wavelength range, which was plagued by line crowding. To2, instead of being a unique cluster, is found to be a normal representative of its class.

Acknowledgements. E.C., S.V. and D.G. gratefully acknowledge support from the Chilean Centro de Astrofísica FONDAP No. 15010003. E.C. and D.G. are also supported by the Chilean Centro de Excelencia en Astrofísica y Tecnologías Afines (CATA).

\section{References}

Alonso, A., Arribas, S., \& Martínez-Roger, C. 1999, A\&AS, 140, 261

Asplund, M., Grevesse, N., \& Sauval, A. J. 2005, in Cosmic Abundances as Records of Stellar Evolution and Nucleosynthesis, ed. T. G. Barnes III, \& F. N. Bash, ASP Conf. Ser., 336, 25

Bellazzini, M., Ibata, R., Monaco, L., et al. 2004, MNRAS, 354, 1263

Brown, J. A., Wallerstein, G., Geisler, D., \& Oke, J. B. 1996, AJ, 112, 1551

Butler, D. J., Martínez-Delgado, D., Rix, H.-W., Peñarrubia, J., \& de Jong, J. T. A. 2007, AJ, 133, 2274

Carney, B. W., Yong, D., Teixera de Almeida, M. L., \& Seitzer, P. 2005, AJ, 130, 1111

Carraro, G., \& Costa, E. 2009, A\&A, 493, 71

Carraro, G., Bresolin, F., Villanova, S., et al. 2004, AJ, 128, 1676

Carraro, G., Vázquez, R. A., Moitinho, A., \& Baume, G. 2005, ApJ, 630, L153

Carraro, G., Geisler, D., Villanova, S., Frinchaboy, P. M., \& Majewski, S. R. 2007, A\&A, 476, 217

Charbonnel, C. 1995, ApJ, 453, L41

Chiappini, C., Matteucci, F., \& Romano, D. 2001, ApJ, 554, 1044

Crane, J. D., Majewski, S. R., Rocha-Pinto, H. J., et al. 2003, ApJ, 594, L119

di Benedetto, G. P. 1998, A\&A, 339, 858

Dinescu, D. I., Martínez-Delgado, D., Girard, T. M., et al. 2005, ApJ, 631, L49

D'Orazi, V., Magrini, L., Randich, S., et al. 2009, ApJ, 693, L31

Friel, E. D., Janes, K. A., Tavarez, M., et al. 2002, AJ, 124, 2693

Frinchaboy, P. M., Majewski, S. R., Crane, J. D., et al. 2004, ApJ, 602, L21

Frinchaboy, P. M., Marino, A. F., Villanova, S., et al. 2008, MNRAS, 391, 39

Grevesse, N., \& Sauval, A. J. 1998, Space Sci. Rev., 85, 161

Ibata, R. A., Irwin, M. J., Lewis, G. F., Ferguson, A. M. N., \& Tanvir, N. 2003, MNRAS, 340, L21

Kupka, F., Piskunov, N., Ryabchikova, T. A., Stempels, H. C., \& Weiss, W. W. 1999, A\&AS, 138, 119

Kurucz, R. L. 1992, in The Stellar Populations of Galaxies, ed. B. Barbuy, \& A. Renzini, IAU Symp., 149, 225

Magrini, L., Sestito, P., Randich, S., \& Galli, D. 2009, A\&A, 494, 95

Marigo, P., Girardi, L., Bressan, A., et al. 2008, A\&A, 482, 883

Marino, A. F., Villanova, S., Piotto, G., et al. 2008, A\&A, 490, 625

Marino, A. F., Milone, A. P., Piotto, G., et al. 2009, A\&A, 505, 1099

Martin, N. F., Ibata, R. A., Bellazzini, M., et al. 2004, MNRAS, 348, 12

Martínez-Delgado, D., Butler, D. J., Rix, H.-W., et al. 2005, ApJ, 633, 205

Mashonkina, L., Gehren, T., Travaglio, C., \& Borkova, T. 2003, A\&A, 397, 275

Mieske, S., Hilker, M., Jordán, A., et al. 2008, A\&A, 487, 921

Milone, A. P., Bedin, L. R., Piotto, G., \& Anderson, J. 2009, A\&A, 497, 755

Moitinho, A., Vázquez, R. A., Carraro, G., et al. 2006, MNRAS, 368, L77

Momany, Y., Zaggia, S. R., Bonifacio, P., et al. 2004, A\&A, 421, L29

Momany, Y., Zaggia, S., Gilmore, G., et al. 2006, A\&A, 451, 515

Newberg, H. J., Yanny, B., Rockosi, C., et al. 2002, ApJ, 569, 245

Pasquini, L., Avila, G., Blecha, A., et al. 2002, The Messenger, 110, 1

Peñarrubia, J., Martínez-Delgado, D., Rix, H. W., et al. 2005, ApJ, 626, 128

Phelps, R. L., Janes, K. A., \& Montgomery, K. A. 1994, AJ, 107, 1079

Piotto, G. 2008, Mem. Soc. Astron. Ital., 79, 334

Ramírez, I., \& Meléndez, J. 2005, ApJ, 626, 465

Randich, S., Bragaglia, A., Pastori, L., et al. 2005, The Messenger, 121, 18

Rocha-Pinto, H. J., Majewski, S. R., Skrutskie, M. F., \& Crane, J. D. 2003, ApJ, 594, L115

Skrutskie, M. F., Cutri, R. M., Stiening, R., et al. 2006, AJ, 131, 1163

Twarog, B. A., Ashman, K. M., \& Anthony-Twarog, B. J. 1997, AJ, 114, 2556

Villanova, S., Piotto, G., King, I. R., et al. 2007, ApJ, 663, 296

Villanova, S., Piotto, G., \& Gratton, R. G. 2009, A\&A, 499, 755

Yanny, B., Newberg, H. J., Grebel, E. K., et al. 2003, ApJ, 588, 824

Yong, D., Carney, B. W., Teixera de Almeida, M. L., \& Pohl, B. L. 2006, AJ, 131,2256 